\title{
Belgeo
}

Revue belge de géographie

$4 \mid 2014$

Miscellaneous

\section{Exploitation minière dans l'arrondissement de Figuil (Cameroun) : problèmes de santé publique et effets environnementaux}

Mining exploitation in the Figuil subdivision (Cameroon): public health problems and environmental impacts

\section{Oumarou Toumba et Anselme Wakponou}

\section{OpenEdition}

Journals

Édition électronique

URL : http://journals.openedition.org/belgeo/14853

DOI : 10.4000/belgeo.14853

ISSN : 2294-9135

Éditeur :

National Committee of Geography of Belgium, Société Royale Belge de Géographie

\section{Référence électronique}

Oumarou Toumba et Anselme Wakponou, « Exploitation minière dans l'arrondissement de

Figuil (Cameroun) : problèmes de santé publique et effets environnementaux », Belgeo [En ligne],

4 | 2014, mis en ligne le 20 décembre 2014, consulté le 19 avril 2019. URL : http://

journals.openedition.org/belgeo/14853; DOI : 10.4000/belgeo.14853

Ce document a été généré automatiquement le 19 avril 2019

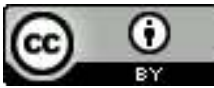

Belgeo est mis à disposition selon les termes de la licence Creative Commons Attribution 4.0 International. 


\title{
Exploitation minière dans
}

l'arrondissement de Figuil (Cameroun) : problèmes de santé publique et effets

\section{environnementaux}

Mining exploitation in the Figuil subdivision (Cameroon): public health problems and environmental impacts

\author{
Oumarou Toumba et Anselme Wakponou
}

\section{Introduction}

1 Les diverses approches scientifiques concernant la grande diversité des interventions humaines et de leurs impacts (intentionnels ou non, aux effets immédiats ou différés) en liaison avec l'extraction des minerais, l'essor industriel, le développement des infrastructures et même les activités de production ne combinent pas toujours les aspects environnementaux et sociaux. C'est ce que nous essayons de faire dans cet article à travers l'analyse intégrée des incidences socio-environnementales de l'exploitation minière dans l'arrondissement de Figuil (figure 1), à travers la méthode de la géographie environnementale. 
Figure 1. Localisation de la zone d'étude.

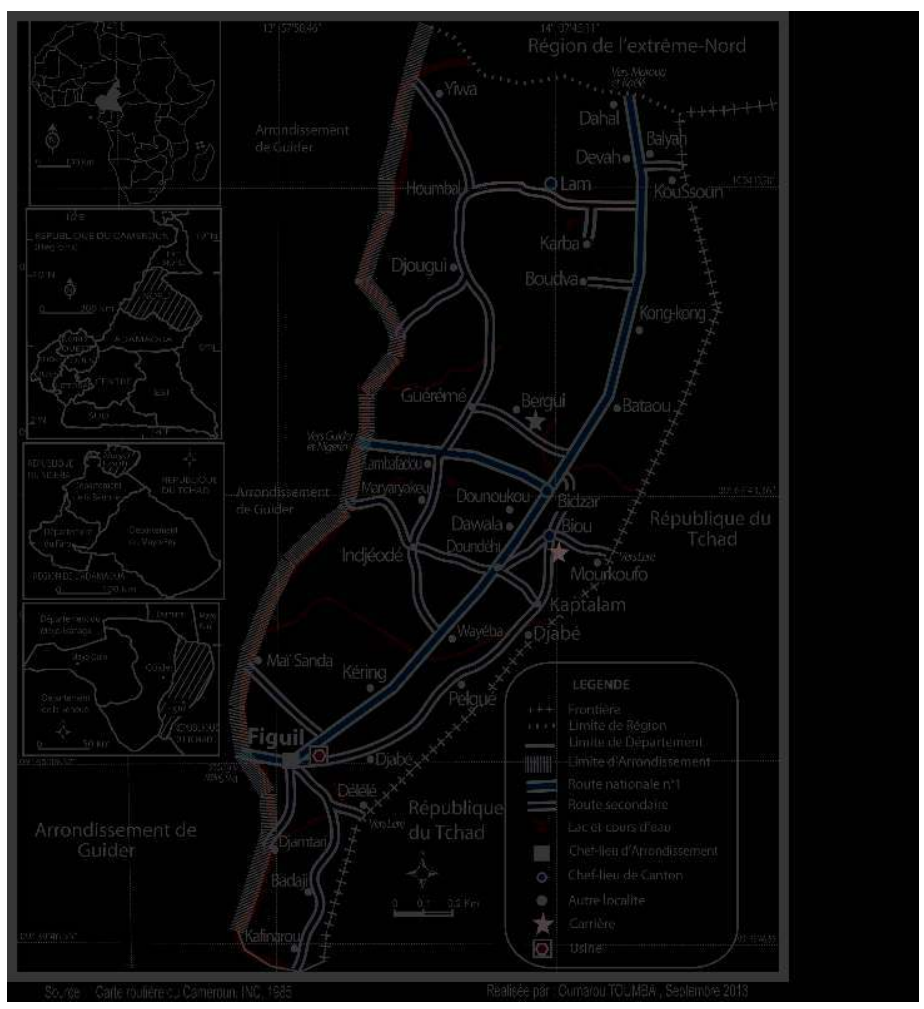

\section{Matériel et méthode}

2 Nous avons procédé à des analyses chimiques de l'eau prélevée des puits et des forages pour déterminer l'origine, la nature et le seuil de la pollution des eaux souterraines des sites d'extraction des marbres et calcaires de Bergui-Bidzar et Biou. Nous avons évalué nos résultats par rapport à la norme de tolérance des substances chimiques dans l'eau de consommation prescrite par l'OMS.

3 Le niveau de pollution atmosphérique a été déterminé en plaçant au sol des sachets en plastique d'un mètre carré de surface, pour une durée de 10 heures par jour, afin de récupérer et de peser (à l'aide d'une balance électronique de marque Stortoriers) la poussière rejetée. Nous avons localisé ces prélèvements respectivement derrière les usines CIMENCAM et Chaux Rocca, ainsi qu'au bord des routes Biou-Bidzar et BerguiBidzar, passages des camions en partance ou en provenance des carrières. Ce travail a été effectué aussi bien en saison sèche (mars 2012 et mars 2013) qu'en saison pluvieuse (août 2013), pendant trois jours successifs de pleine activité d'extraction minière (mardi, mercredi et jeudi).

4 Pour relever le taux de pollution au sein de l'usine, nous avons utilisé l'appareil Dustcan Scout. Pour l'opération, le stylet capteur de ce dernier, tenu en main, enregistre automatiquement toutes les dix secondes la concentration de poussières. Les résultats des différentes mesures sont présentés sur des graphes qui montrent l'évolution de la concentration de poussière. Nous avons comparé nos résultats à ceux obtenus par Watio Zebabou (2008). 
5 Pour les enquêtes à Bergui et à Biou, nous avons utilisé la méthode du focus group discussion. Nous avons corrélé le résultat de ces enquêtes au dépouillement des archives sanitaires du dispensaire de Bidzar et de l'Hôpital de district de Figuil. Le but a été de recenser les maladies liées à l'extraction du calcaire dont souffrent les populations riveraines. À l'aide du logiciel de statistiques SPSS 10.0, nous avons construit des graphes qui montrent l'évolution du pourcentage de malades de 1987 à 2013 et la corrélation entre l'extraction de calcaire et le taux de malades.

6 Nous avons également fait un inventaire des œuvres sociales menées par les entreprises minières, pour apprécier les retombées de l'exploitation minière en faveur de la protection de l'environnement.

\section{Résultats}

\section{Description du secteur de l'extraction minière à Figuil}

7 Les activités du secteur minier dans l'arrondissement de Figuil portent sur l'extraction des ressources de construction. Il s'agit des carrières (calcaire/marbre, sable, argile et granulats) et des usines de transformation de ces matériaux. Ce secteur compte deux entreprises, dont Chaux Rocca (ROCAGLIA), qui possède une usine à Figuil et une carrière à Biou. Celle-ci exploite le marbre et le granulat pour la fabrication du gravier de revêtement de la chaussée, des carreaux de marbre, de la chaux vive et de la chaux agricole. L'autre entreprise, $\mathrm{CIMENCAM}^{1}$, exploite trois carrières (argile, sable et calcaire) pour la fabrication du ciment $\mathrm{CPJ} 35$ et CPJ40. D'autres entreprises minières sont en attente d'agrément pour y exercer leur activité.

Le secteur minier contribue pour beaucoup à l'économie locale et nationale. La redevance minière a engendré au cours de l'exercice 2009 à 2011, respectivement 85930656 FCFA en 2009, 90290689 FCFA en 2010 et 103117037 FCFA en 2010 (ITIE Cameroun, 2013)2. L'industrie extractive fournit près de 500 emplois dans l'arrondissement de Figuil et supporte plus d'une dizaine de sous-traitants.

9 Le nouveau code minier camerounais est attractif du fait du soutien offert par l'État camerounais, ainsi que par des institutions financières internationales, aux sociétés qui y investissent. Il offre des allègements des taxes et des procédures. Le fait d'investir davantage dans ce secteur entraine, pour les entreprises minières, le gouvernement camerounais et les collectivités locales, une gamme de défis socio-environnementaux, alors que les capacités de gouvernance en matière d'environnement sont faibles. En outre, la collectivité locale de Figuil ne dispose pas de ressources et des compétences nécessaires pour contrôler efficacement les incidences socio-environnementales des activités du secteur extractif et les risques écologiques qui exacerbent les impacts de la fragilité de l'environnement local.

\section{Exploitation minière, une action exacerbant la fragilité d'une aire sèche}

10 L'exacerbation de la fragilité écologique se traduit par la pollution de l'air, des eaux souterraines et la détérioration du couvert végétal. 


\section{La pollution de l'air atmosphérique et ses incidences sanitaires}

11 La situation de la pollution atmosphérique dans l'arrondissement de Figuil peut être assimilée au phénomène de bumbing qui fabrique plusieurs millions de tonnes d'aérosols par an à la suite des émissions de poussières des carrières de Bergui-Bidzar et de Biou, et des usines de CIMENCAM et Chaux Rocca (ROCAGLIA) à Figuil. Ces poussières sont des particules d'origine minérale émises d'une part lors du concassage des blocs de calcaire et de leur transport par trémie vers la zone de chargement des camions de transport à l'usine et, d'autre part, lors des opérations de fabrication des carreaux de marbre, du ciment et de la chaux dans les usines (figure $2 \mathrm{a}, \mathrm{b}, \mathrm{c}$ ).

Figure $2 \mathrm{a}, \mathrm{b}, \mathrm{c}$. Quelques modalités de rejet et de soulèvement de la poussière.
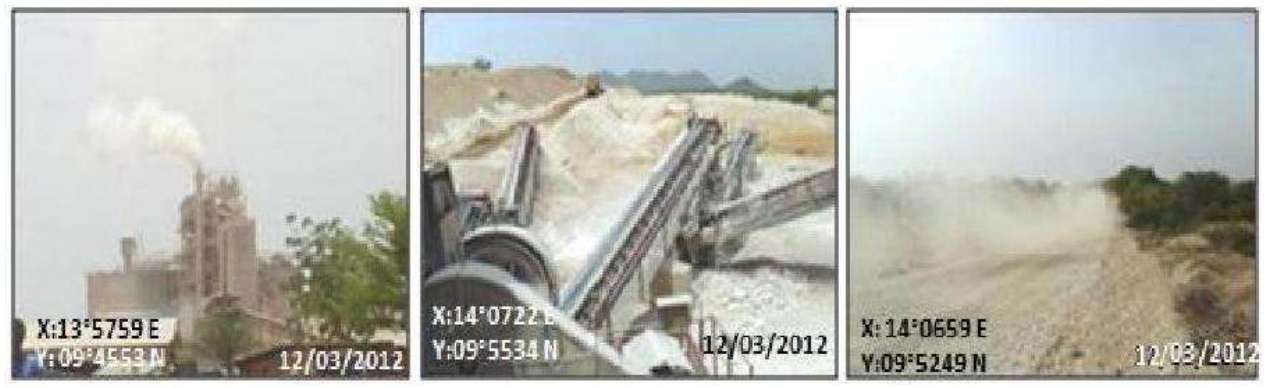

Émission de la poussière et de la fumée par la cheminée de l'usine CIMENCAM à Figuil. Émission de la poussière à la trémie de la carrière de Bergui.

Émission de la poussière au passage des camions sur la route de la carrière de Biou.

Source : Cliché Oumarou Toumba

12 La cimenterie et la marbrerie de Figuil émettent dans l'atmosphère du silicate issu des calcaires et marbres. Ces émissions ont été évaluées respectivement à plus de $200 \mu \mathrm{g} / \mathrm{m}^{3}$ par jour pendant les saisons sèches de mars 2012 et 2013 et à plus de $100 \mu \mathrm{g} / \mathrm{m}^{3}$ pendant la saison pluvieuse du mois d'août 2013 aux alentours des usines CIMENCAM, Chaux Rocca et des routes menant aux carrières.

13 Si la concentration est sensiblement égale durant les trois jours de mesure, elle varie cependant d'une zone à l'autre (tableau 1, figure 3).

Tableau 1. Mesures de concentration de poussière dans les zones minières de Bidzar-Figuil.

\begin{tabular}{|c|c|c|c|c|c|c|c|c|c|}
\hline \multirow[b]{2}{*}{ Zone mesurée } & \multicolumn{3}{|c|}{ Mars 2012} & \multicolumn{3}{|c|}{ Mars 2013} & \multicolumn{3}{|c|}{ Août 2013} \\
\hline & $\begin{array}{l}\text { ler jour } \\
\left(\mu \mathrm{g} / \mathrm{m}^{3}\right)\end{array}$ & $\begin{array}{l}2 \mathrm{e} \text { jour } \\
\left(\mu \mathrm{g} / \mathrm{m}^{3}\right)\end{array}$ & $\begin{array}{l}\text { 3e jour } \\
\left(\mu \mathrm{g} / \mathrm{m}^{3}\right)\end{array}$ & $\begin{array}{l}\text { 1er jour } \\
\left(\mu \mathrm{g} / \mathrm{m}^{3}\right)\end{array}$ & $\begin{array}{l}\text { 2e jour } \\
\left(\mu \mathrm{g} / \mathrm{m}^{3}\right)\end{array}$ & $\begin{array}{l}\text { 3e jour } \\
\left(\mu \mathrm{g} / \mathrm{m}^{3}\right)\end{array}$ & $\begin{array}{l}\text { 1er jour } \\
\left(\mu \mathrm{g} / \mathrm{m}^{3}\right)\end{array}$ & $\begin{array}{l}2 \mathrm{e} \text { jour } \\
\left(\mu \mathrm{g} / \mathrm{m}^{3}\right)\end{array}$ & $\begin{array}{l}3 \mathrm{e} \text { jour } \\
\left(\mu \mathrm{g} / \mathrm{m}^{3}\right)\end{array}$ \\
\hline Route Bergi-Bidzar & 340,3 & 320,4 & 320 & 341,4 & 323,1 & 324,3 & 101,7 & 143,2 & 203,3 \\
\hline Route Biou-Bidzar & 220,5 & 240,4 & 220 & 227,5 & 240,1 & 234 & 115,6 & 117,3 & 121,8 \\
\hline $\begin{array}{l}\text { Alentours } \\
\text { CIMENCAM }\end{array}$ & 230,5 & 210,8 & 230 & 221,5 & 207,8 & 223,2 & 118,3 & 103,9 & 106,4 \\
\hline Alentours Chaux Roca & 340,5 & 320,8 & 350 & 310,5 & 310,8 & 310,3 & 145,2 & 146,1 & 144,7 \\
\hline
\end{tabular}

Source : enquêtes de terrain, 2012 et 2013 
Figure 3. Répartition de trois jours de concentration de poussières par zone.

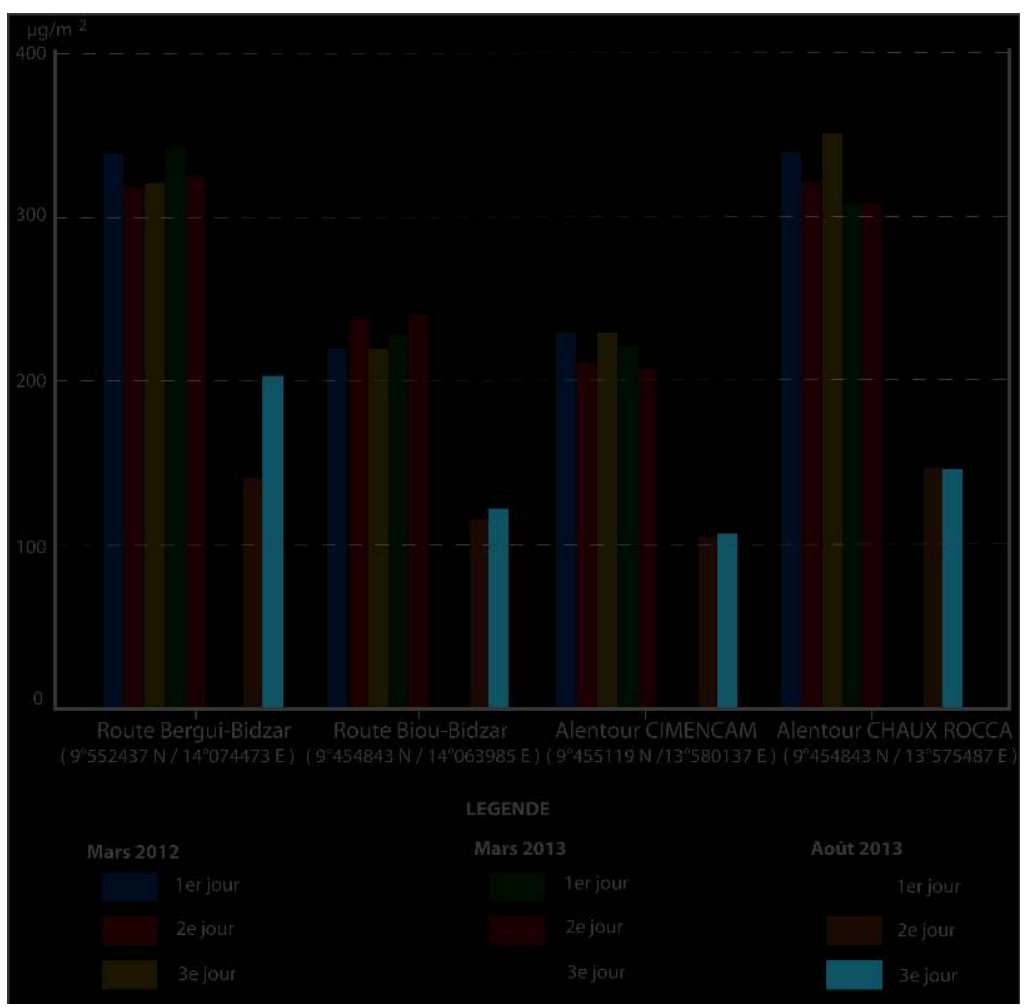

En mars 2012 et 2013, les alentours de l'usine Chaux Rocca et la route Bergui-Bidzar étaient les plus poussiéreux (plus de $300 \mu \mathrm{g} / \mathrm{m}^{3}$ ), même si en mars 2013 nous avons observé une légère baisse. Celle-ci était due à un début d'application de mesure de contrôle environnemental par la société Chaux Rocca. En effet, en août 2013, nous avons enregistré, en général, moins de $200 \mu \mathrm{g} / \mathrm{m}^{3}$. Plus marquant, sur la route Bergui-Bidzar, au premier jour de mesure, la concentration de poussière a été de $101,7 \mu \mathrm{g} / \mathrm{m}^{3}$. Ce faible taux s'explique peut-être par la pluie tombée au petit matin.

15 En 2008, Watio Zebabou, en 4 minutes, avait enregistré à la bande d'entrée des broyeurs une concentration de $1438 \mu \mathrm{g} / \mathrm{m}^{3}$ et de $249 \mu \mathrm{g} / \mathrm{m}^{3}$ au pied de la trémie crue (figures 4 et 5). Ces mesures révèlent que l'origine du rejet de la poussière se trouve à l'intérieur de l'usine et les sources de pollution sont multiples.

16 Nos mesures et celles de Watio Zebabou (2008) prouvent donc que la concentration de poussières est deux fois plus élevée que la moyenne annuelle $\left(80 \mu \mathrm{g} / \mathrm{m}^{3}\right)$ ou la moyenne journalière $\left(260 \mu \mathrm{g} / \mathrm{m}^{3}\right)$ prescrite par les Normes Environnementales et procédure d'inspection au Cameroun. 
Figure 4. Concentration de poussières totales aux bandes d'entrée des broyeurs 1 et 2, côté tour.

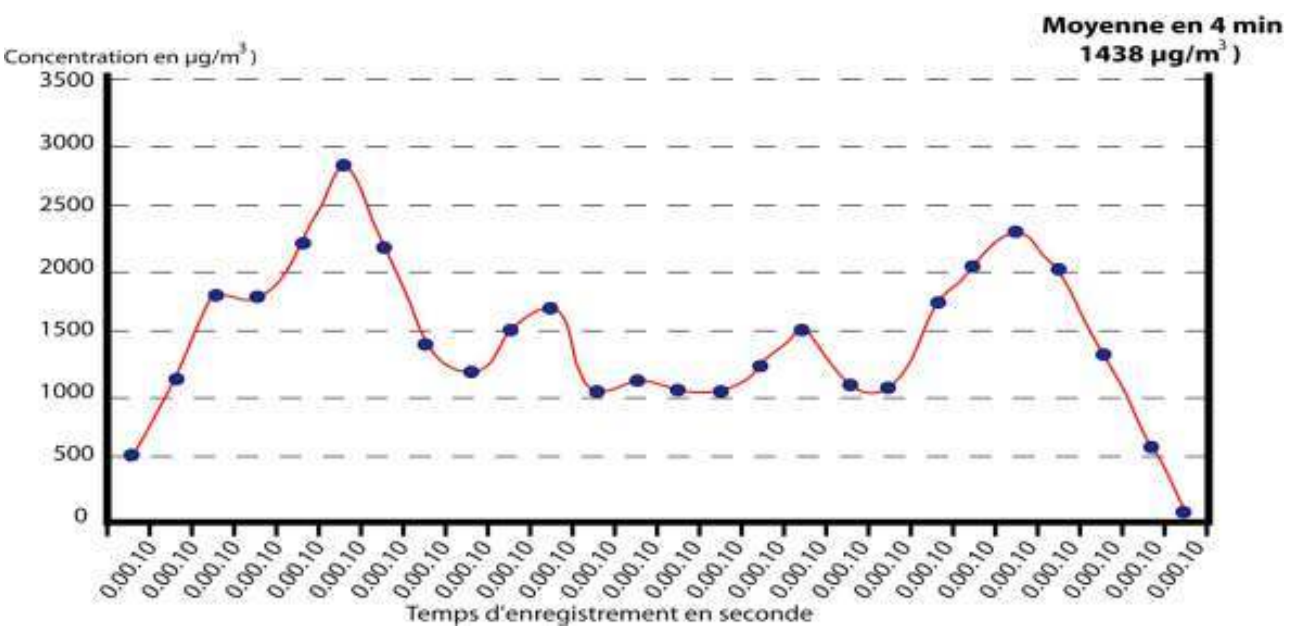

Source : Watio, 2008

Figure 5. Concentration de poussière au pied de la trémie crue.

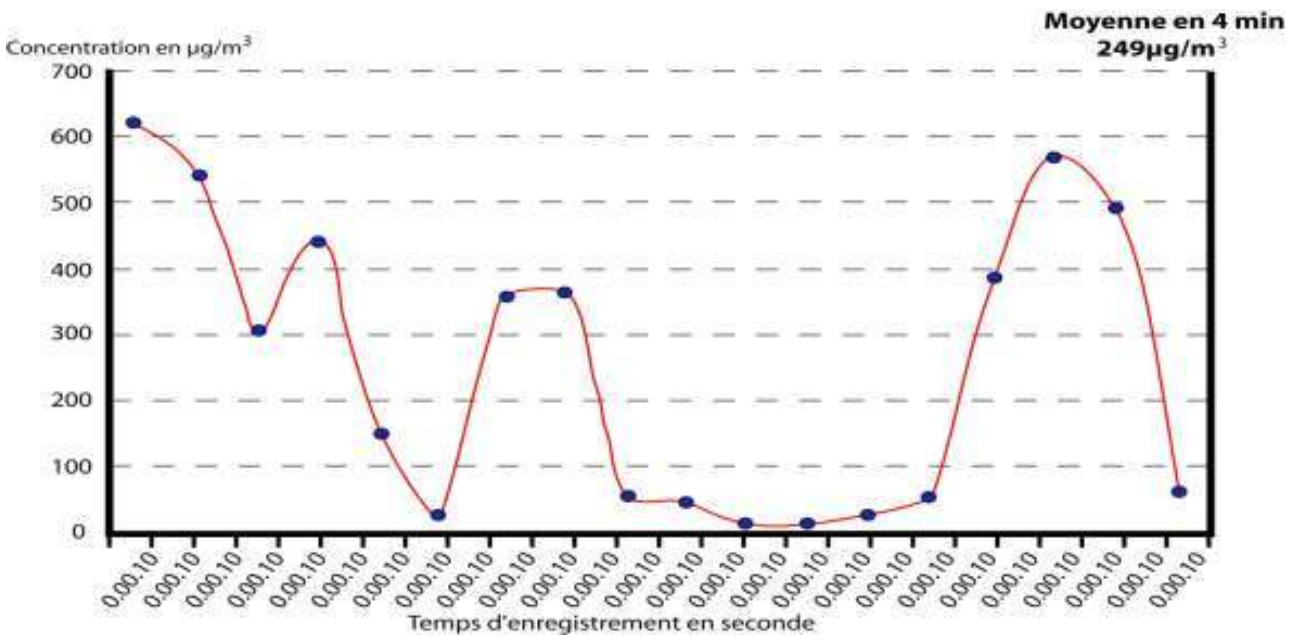

Source : Watio, 2008

17 Les poussières ainsi rejetées ont des influences néfastes sur l'organisme humain. Elles justifient l'état morbide de la population riveraine. En effet, quatre malades sur dix souffrent de maladies pulmonaires (la pneumonie, la bronchite, l'asthme, la tuberculose et la rhynobronchite) $)^{3}$. Depuis 2011, plus de $20 \%$ des malades souffrent de problèmes respiratoires (tableau 2). Ces infections sont dues à l'absorption des plus fines particules des poussières $(<2 \mu \mathrm{m})$ qui pénètrent dans les alvéoles pulmonaires (Renoux, 1984 ; Niessner, 1986; Almeida, 1991 et Jaenicke, 1993). Notons que par le passé (années 1980-90), ces maladies apparaissaient surtout en saison sèche, alors que nos jours on note une véritable endémie, toutes saisons confondues (tableau 2). 
Tableau 2. Taux de la population (\%) souffrant des maladies respiratoires de 1987 à 2012, par mois.

\begin{tabular}{|c|c|c|c|c|c|c|c|c|c|c|c|c|c|}
\hline nnées & Janvier & Février & Mars & Avril & Mai & Juin & Juillet & Août & Sept. & Oct. & Nov. & Déc. & Moy.An \\
\hline 1987 & 12 & 11,7 & 11 & 6 & 3 & 0,4 & 0,3 & 0,1 & 1 & 0,2 & 1 & 7 & 4,5 \\
\hline 1988 & 11 & 11 & 9 & 13 & 3 & 0,5 & 0,8 & 0,2 & 1 & 1 & 7 & 9 & 5,5 \\
\hline 1989 & 13 & 15,4 & 13 & 8 & 6 & 4 & 1 & 0,1 & 0,3 & 0,8 & 1 & 14 & 6,4 \\
\hline 1990 & $?$ & $?$ & $?$ & $?$ & $?$ & $?$ & $?$ & $?$ & $?$ & 7 & 9 & 11 & 2,3 \\
\hline 1991 & 12 & 15 & 11,6 & 12 & 9 & 5 & 0,6 & 0,2 & 1 & 8 & 10 & 13 & 8,1 \\
\hline 1992 & 14 & 13 & 12 & 11 & 7 & 9 & 5 & 3 & 6 & 12 & 17 & 15 & 10,3 \\
\hline 1993 & 11,5 & 16,6 & 14 & 15 & 11 & 6 & 6 & 1 & 9 & 11 & 11 & 16 & 10,7 \\
\hline 1994 & 17,6 & 16 & 13 & 14 & 12 & 9 & 6 & 3 & 11 & 13 & 16 & 16,4 & 12,3 \\
\hline 1995 & 14 & 17 & 14 & 12,5 & 12 & 11 & 6,6 & 9 & 11 & 14 & 14 & 16,6 & 12,6 \\
\hline 1996 & 13 & 14 & 13 & 11,6 & 10 & 9 & 5 & 13 & 13,6 & 13 & 15 & 18 & 12,4 \\
\hline 1997 & 15 & 14,7 & 14 & 11 & 7 & 10 & 8,3 & 9 & 14,7 & 14,7 & 16 & 17 & 12,6 \\
\hline 1998 & 18 & 16 & 15 & 13 & 11 & 11 & 8,5 & 10 & 15,4 & 16 & 16,7 & 19 & 14 \\
\hline 1999 & 21 & 18 & 17 & 14 & 12 & 12,5 & 9,6 & 10,3 & 16 & 17,8 & 17 & 20,7 & 15,5 \\
\hline 2000 & 22 & 17 & 17 & 13 & 10 & 13 & 10,3 & 11 & 15,8 & 18 & 19,4 & 21 & 15,6 \\
\hline 2001 & 23 & 20 & 15 & 14,2 & 11 & 12 & 11,6 & 10,6 & 16,3 & 18,4 & 18 & 19 & 16 \\
\hline 2002 & 25 & 21 & 17 & 14,7 & 12 & 11,4 & 12 & 11,4 & 16 & 19 & 21 & 23 & 17 \\
\hline 2003 & 16 & 17 & 12,4 & 12 & 10 & 8 & 9 & 6 & 14 & 17 & 16,8 & 17 & 13 \\
\hline 2004 & 27 & 25 & 24 & 15 & 16 & 12 & 11 & 10,4 & 16,4 & 17,6 & 20,3 & 20 & 18 \\
\hline 2005 & 28 & 25,7 & 23 & 22 & 14 & 16 & 11,3 & 10 & 16,6 & 17 & 21 & 22 & 18,8 \\
\hline 2006 & 26 & 21 & 22,7 & 21 & 16 & 13,7 & 10,6 & 9 & 15,4 & 17,4 & 26 & 24,4 & 18,6 \\
\hline 2007 & 27 & 25,4 & 23 & 20 & 15 & 13 & 11,8 & 11 & 16 & 17 & 21,5 & 23,8 & 18,7 \\
\hline 2008 & 28 & 23 & 21 & 21 & 16 & 18 & 12,5 & 11,4 & 17,7 & 18 & 22 & 24 & 19,4 \\
\hline 2009 & 26 & 25 & 23,6 & 23 & 15,6 & 15 & 12,8 & 11,6 & 17,3 & 18,3 & 21 & 23,7 & 19,4 \\
\hline 2010 & 28 & 28,6 & 25 & 23,8 & 17,7 & 14 & 13,4 & 12,7 & 17,5 & 18,7 & 21,4 & 24,6 & 20,5 \\
\hline 2011 & 26 & 28 & 27 & 23 & 17 & 15 & 13 & 12,6 & 17,8 & 19 & 21 & 24 & 20,3 \\
\hline 2012 & 26,8 & 27 & 26 & 23,4 & 18 & 14,6 & 15 & 12 & 17 & 19,7 & 21,3 & 25 & 20,5 \\
\hline
\end{tabular}

? : Données inexistantes

La figure 6 présente l'évolution du taux de malades de 1987 à 2012, dans l'arrondissement de Figuil. De 1987 à 1994, en saison sèche (de septembre à mai), les formations sanitaires ont enregistré plus de malades qu'en saison pluvieuse (de juin à août), au total 2956 malades $^{4}$, soit un taux relatif de $9 \%$. De 1995 à 2002, la situation s'aggrave : 6174 malades ont été enregistrés, soit un taux de 14,5\%. Mais c'est durant la période allant de 2003 à 2012 que la crise sanitaire s'affirme. En saison pluvieuse comme en saison sèche, le taux moyen de malades est de $20 \%$. 
Figure 6. Évolution du taux de malades au sein de la population de 1987 à 2012.

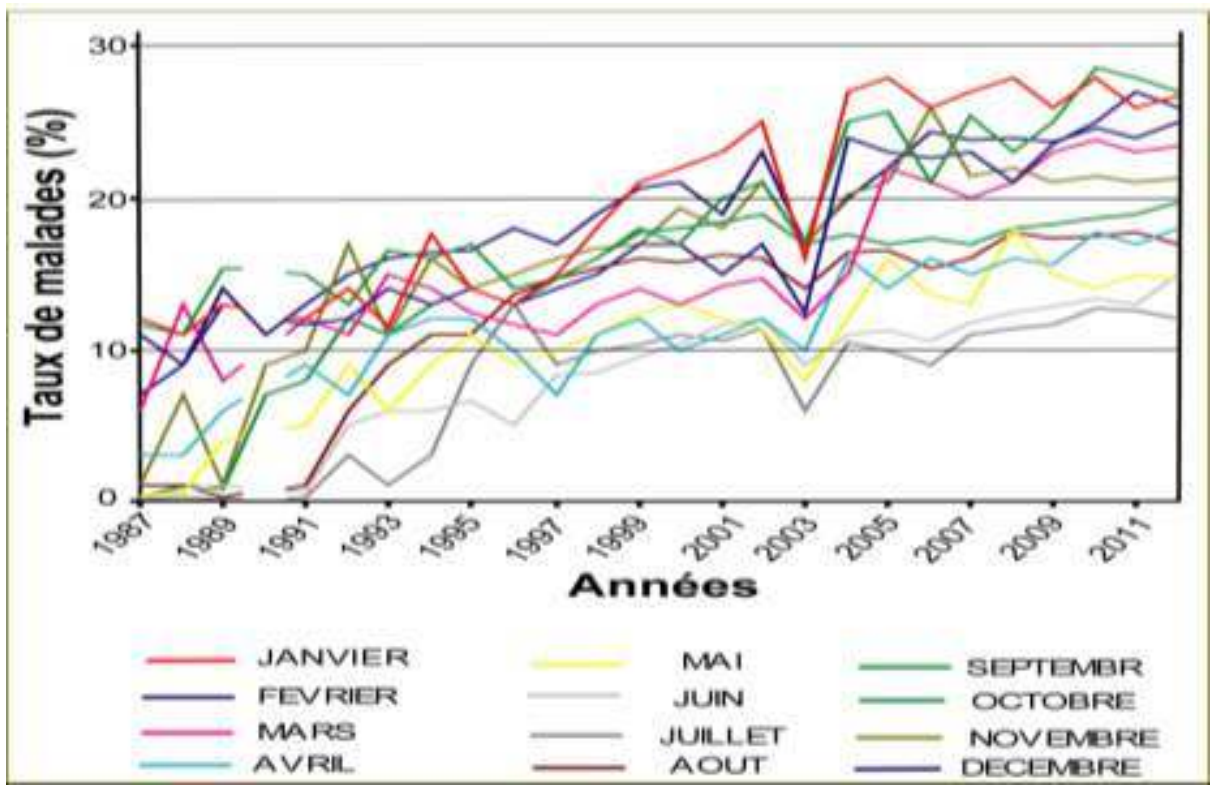

19 L'accroissement considérable du nombre de malades respiratoires est étroitement lié à l'exploitation minière: une corrélation significative et forte $(0,89)$ relie le nombre de malades à l'évolution de l'activité minière (tableau 3 et figure 7).

Tableau 3. Exploitation du calcaire en tonnes et nombre de malades par an de 1987 à 2012.

\begin{tabular}{|c|c|c|c|c|c|}
\hline Annees & $\begin{array}{l}\text { Exploitation } \\
\text { de calcaire } \\
\text { (Uan) }\end{array}$ & $\begin{array}{c}\text { Malades } \\
\text { par an }\end{array}$ & Annes & $\begin{array}{l}\text { Exploitation } \\
\text { de calcaire } \\
\text { (t/an) }\end{array}$ & $\begin{array}{c}\text { Malades } \\
\text { par an }\end{array}$ \\
\hline 1987 & $?$ & 1576 & 1996 & $?$ & 5455 \\
\hline 1988 & $?$ & 2027 & 1997 & $?$ & 5722 \\
\hline 1989 & $?$ & 2390 & 1998 & $?$ & 6554 \\
\hline 1990 & $?$ & 859 & 1999 & $?$ & 7323 \\
\hline 1991 & $?$ & 3159 & 2000 & 153122 & 7585 \\
\hline 1992 & $?$ & 4127 & 2001 & 156863 & 7801 \\
\hline 1993 & $?$ & 4361 & 2002 & 138129 & 3502 \\
\hline 1994 & $?$ & 5149 & 2003 & 118363 & 1503 \\
\hline 1995 & $?$ & 5453 & 2004 & 162461 & 9469 \\
\hline
\end{tabular}

? : Données inexistantes 
Figure 7. Courbes d'évolution de la production du calcaire et du nombre de malades.

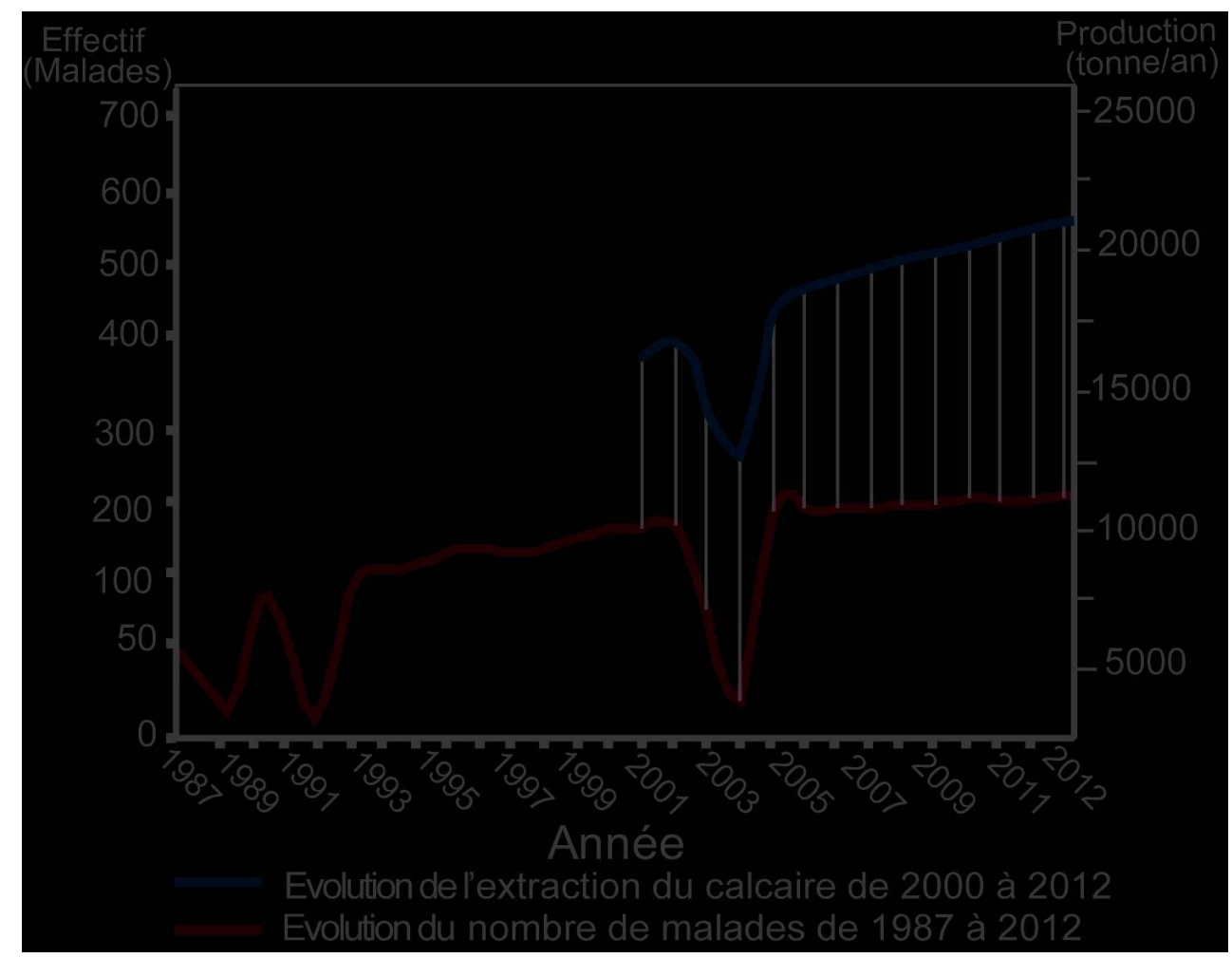

Les poussières émises par les carrières nuisent au vécu quotidien des populations riveraines. Leur peau blanchit à cause de dépôts de poudre de calcaire. Ils ont du mal à faire la sieste à l'ombre des arbres et à y prendre des repas communautaires. En effet, le vent dépose la poudre de calcaire sur les nattes étalées, sur les plats de nourriture et les réserves d'aliments (sorgho, arachides, légumes, etc.). L'incidence quotidienne de la pollution de l'air est donc réelle. Celle de l'eau n'est pas non plus négligeable.

\section{La pollution chimique des eaux souterraines et les méfaits gastro-entériques}

21 L'analyse des caractéristiques chimiques des eaux de Bergui et Biou, révèle que les nappes phréatiques sont chargées d'acide carbonique à plus de $250 \mathrm{mg} / \mathrm{l}$ (tableau 4).

Tableau 4. Résultats de l'analyse chimique de l'eau.

\begin{tabular}{|l|c|c|c|c|c|c|c|c|c|c|c|}
\hline Échantillons & $\begin{array}{c}\text { Coordonnées } \\
\text { géographiques } \\
(\mathrm{X} / \mathrm{Y} / \mathrm{Z})\end{array}$ & $\begin{array}{c}\mathrm{Na}^{+} \\
(\mathrm{mg} / 1)\end{array}$ & $\begin{array}{c}\mathrm{K}^{+} \\
(\mathrm{mg} / 1)\end{array}$ & $\begin{array}{c}\mathrm{Ca}_{2}^{+} \\
(\mathrm{mg} / 1)\end{array}$ & $\begin{array}{c}\mathrm{Mg}_{2}^{+} \\
(\mathrm{mg} / 1)\end{array}$ & $\begin{array}{c}\mathrm{NH}_{4}^{+} \\
(\mathrm{m} / 1)\end{array}$ & $\begin{array}{c}\mathrm{HCO}_{3}^{-} \\
(\mathrm{mg} / 1)\end{array}$ & $\begin{array}{c}\mathrm{NO}_{3}^{-} \\
(\mathrm{mg} / 1)\end{array}$ & $\begin{array}{c}\mathrm{SO}_{4}{ }^{2-} \\
(\mathrm{mg} / 1)\end{array}$ & $\begin{array}{c}\mathrm{Cl}^{-} \\
(\mathrm{mg} / 1)\end{array}$ & $\begin{array}{c}\mathrm{HPO}_{4}{ }^{2-} \\
(\mathrm{mg} / 1)\end{array}$ \\
\hline Puits Biou & $\begin{array}{c}09^{\circ} 52^{\prime} 12 / 14^{\circ} 06^{\prime} 28 \\
405 \mathrm{~m}\end{array}$ & 0,22 & 1,74 & 13,51 & 7,84 & 0 & 294,02 & 16,05 & 4,24 & 3,8 & 0 \\
\hline $\begin{array}{l}\text { Forage } \\
\text { Bergui }\end{array}$ & $\begin{array}{c}09^{\circ} 55^{\prime} 19 / 14^{\circ} 07^{\prime} 26 \\
405 \mathrm{~m}\end{array}$ & 0,11 & 0 & 18,02 & 10,93 & 0 & 375,76 & 11,51 & 18,78 & 2,2 & 0 \\
\hline $\begin{array}{l}\text { Forage } \\
\text { Bergui }\end{array}$ & $\begin{array}{c}09^{\circ} 55^{\prime} 36 / 14^{\circ} 07^{\prime} 41 \\
422 \mathrm{~m}\end{array}$ & 0,26 & 0 & 13,51 & 20,68 & 0 & 463,6 & 1,71 & 9,44 & 1,6 & 0 \\
\hline Puits Bergui & $\begin{array}{c}09^{\circ} 55^{\prime} 26 / 14^{\circ} 07^{\circ} 09 \\
399 \mathrm{~m}\end{array}$ & 0,41 & 0,16 & 18,02 & 20,41 & 0 & 439,2 & 10,33 & 13,04 & 1,8 & 0 \\
\hline
\end{tabular}

Source : Analyse chimique de l'eau, IRAD Ekona (région du Sud-ouest), juillet 2012.

22 Ces nappes, souvent à seulement $1 \mathrm{~m}$ sous la carrière de Bergui en saison pluvieuse, subissent la «nitrification» due à l'utilisation du nitrate mélangé au sygmagel et au nitram $09(70 / 200)$ dans les explosifs qui servent à l'abattage des roches (environ $50 \mathrm{~kg}$ à 
chaque opération et environ 2500 tonnes par $\left.\mathrm{an}^{5}\right)$. Du fait de l'accroissement de l'extraction minière, les doses de nitrate dépasseront la norme exigée par l'OMS (50 mg/l) si elles ne sont pas contrôlées. Déjà, ces eaux sont de qualité moindre (classe $1 \mathrm{~B})^{6}$, mais satisfont à tous les usages domestiques des populations. L'excès du nitrate, selon Veyret et Pech (1993), entraîne la morbidité de la population en général et, en particulier, des troubles graves voire la mort de nourrissons. Aussi, conviendrait-il d'intégrer le problème des nappes et de leur qualité dans la planification urbaine (plans locaux d'urbanisme, cartes communales...), afin d'assurer l'accès à l'eau au standard mondial et en même temps pérenniser la ressource végétale qui en dépend.

\section{La forte pression de l'exploitation minière sur des ressources végétales indigentes} milieu qui, par nature, est clairsemé d'arbustes, donc fragile (Wakponou et al., 2007). « La société Chaux Rocca qui est la plus grande entreprise consommatrice de bois de la localité, devrait s'investir davantage dans l'activité forestière pour pérenniser cette ressource qui lui est capitale » (Oumarou Toumba, 2009). Tel n'est pourtant pas le cas, bien qu'elle ait une pépinière à Figuil. Ses deux zones de reboisement (Mayo Kéwé entre $9^{\circ} 51^{\prime} 25.33$ et $9^{\circ} 51^{\prime} 27.18 \mathrm{~N}$ et $14^{\circ} 02^{\prime} 27.65$ et $14^{\circ} 02^{\prime} 30.00 \mathrm{E}$ et Bafouni entre $9^{\circ} 44^{\prime} 06.54$ et $9^{\circ}$ $44^{\prime} 14.82 \mathrm{~N}$ et $13^{\circ} 58^{\prime} 05.59$ et $\left.13^{\circ} 58^{\prime} 15.57 \mathrm{E}\right)$ ont moins de dix hectares ${ }^{7}$ chacune et ne compensent pas la coupe de bois de chauffe ( 1 hectare par coupe quotidienne, soit un volume de $\left.5706 \mathrm{~m}^{3} / \mathrm{an}\right)^{8}$. Ces aires ne sont pas bien aménagées et entretenues. Pourtant, la convention de Rio de Janeiro de 1992, ratifiée par le Cameroun, stipule que : « où un arbre est coupé, on doit en planter dix afin de pérenniser la ressource ».

La législation en vigueur, qui interdit la coupe des espèces ligneuses ayant une valeur sociale, n'est pas respectée. C'est pourquoi Toumba Tizi (2003) évoque les vieillards qui s'inquiètent du manque de respect pour certaines espèces ligneuses (Tamarindus indica, Ficus platyphylla, Ficus sp., Adansonia digitata) qui sont réputées abriter des esprits bienfaiteurs ou malfaiteurs en pays Guidar. On observe les traces de coupes à blanc des arbres de toute espèce et de tout âge, même dans les forêts-galeries qui bordent les cours d'eau.

Figure $8 \mathrm{a}$, b. Exploitation irrationnelle du bois pour la cuisson du marbre à Chaux Rocca (ROCAGLIA).
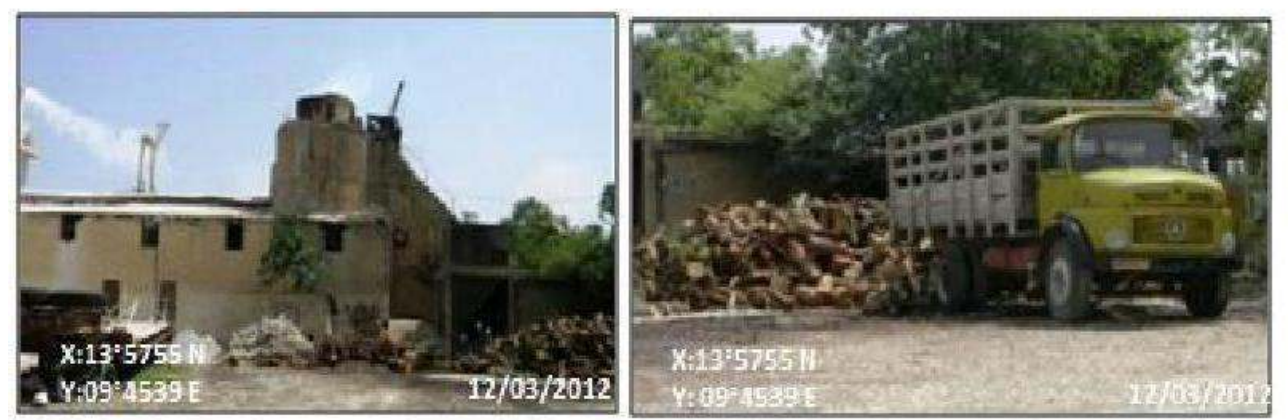

Source : Cliché Oumarou Toumba 
gure 8a montre un four traditionnel de cuisson du marbre pour la fabrication de la chaux vive. Ce four fonctionne au bois de chauffe dont les fagots frais en séchage sont transportés quotidiennement par un camion de $12 \mathrm{~m}^{3}$ (figure $8 \mathrm{~b}$ ).

\section{Discussion}

Les résultats de notre étude révèlent que l'exploitation minière exacerbe la fragilité d'une aire sèche. Cette exacerbation se traduit par la pollution de l'air due au rejet de la calcite lors du concassage, du transport et de la calcination à l'usine ; et par la nitrification des nappes phréatiques par des résidus des produits explosifs de l'abattage des roches. La pollution atmosphérique est évaluée à plus de $200 \mu \mathrm{g} / \mathrm{m}^{3}$ en $10 \mathrm{~h}$. Ce résultat, à l'exemple de celui obtenu par Watio Zebabou (figure 5), témoigne que la pollution, par le rejet de la poussière, dépasse le seuil de tolérance de la norme environnementale en vigueur au Cameroun, qui est de $80 \mu \mathrm{g} / \mathrm{m}^{3}$ en $24 \mathrm{~h}$.

études réalisées par le Bureau des Recherches Géologiques et Minière (BRGM) dans le domaine du forage pour déterminer le volume du calcaire à Bergui et celles faites par Galdibé (2008) et Watio (2008) ont suggéré de mener une étude sur l'impact de l'activité minière sur les eaux souterraines car, en saison pluvieuse, la nappe phréatique se situe à $1 \mathrm{~m}$ sous la carrière de Bergui ${ }^{9}$. À cet effet, les analyses chimiques que nous avons effectuées répondent à ces souhaits.

Cette étude aboutit au même résultat qu'une autre faite par Ngounou Ngatcha et al. (2000) sur la pollution par les nitrates des aquifères du Quaternaire de la partie septentrionale du Cameroun. Ainsi, le résultat de notre étude confirme que l'eau est «la victime de l'exploitation minière » (Lyon, 1997).

Les entreprises lésinent sur le respect des droits de l'environnement. Les actions sociales qu'elles mènent sont mitigées. En effet, elles ne s'impliquent pas véritablement dans la gestion environnementale pour protéger le couvert végétal indigent, ni comme acteurs de premier ordre dans l'aménagement urbain de Figuil. En outre, ces entreprises travaillent le plus souvent seules, au lieu de décider et d'agir en partenariat avec les acteurs locaux (autorités publiques et communautés riveraines des sites miniers). « Il n'y a pas de coordination avec les acteurs sociaux de la localité pour propulser le développement local », comme l'a montré ailleurs Alejandro Sánchez ${ }^{10}$, cité par Lemieux (2010). Or «La protection de l'environnement doit rester le but premier de la société même si cela signifie une production minière réduite. L'adoption de ce bon comportement nous permettra de ne pas transformer la course à l'extraction (...) en cauchemar de l'environnement empoisonné » (De Rosa \& Lyon, 1997).

Par ailleurs, il est difficile d'évaluer, de façon quantitative, les préoccupations relatives aux répercussions des opérations extractives sur les droits de l'environnement ainsi que les défis sous-jacents. C'est ce qui a inspiré la déclaration de Sherrington en 2008, disant que: «Le secteur minier industriel ne devrait pas espérer résoudre le problème, mais apprendre à gérer le défi ». Ainsi, les sociétés minières de Figuil qui auront la réputation de respecter les normes de RSE, et même d'aller au-delà de celles-ci, pourront réussir sur le plan économique. En effet, les performances sur le plan social et environnemental des industries extractives auront un impact positif sur le développement local durable. Cela ne peut être possible qu'en prenant en compte le long terme et la gestion raisonnée des ressources et de l'environnement dans un processus d'accroissement des richesses. 


\section{Conclusion}

Il ressort de cette étude que l'exploitation minière présente des risques écologiques majeurs au Cameroun. En effet, l'aire fragile de Figuil est victime de la coupe abusive du bois de chauffe, de la pollution de l'air par la calcite, de la nitrification de ses nappes phréatiques et d'une exacerbation de la morbidité des populations. En outre, l'idée du nouveau code minier camerounais de 2001 de juguler ou d'atténuer les effets néfastes de l'extraction minière et d'offrir des compensations aux populations riveraines n'est pas véritablement mise en pratique. Une gestion efficiente de l'environnement n'est toujours pas à l'ordre du jour. C'est pourtant la voie nécessaire pour que le secteur minier puisse contribuer à la protection des écosystèmes et à l'amélioration du cadre de vie des populations actuelles et futures, dans une vision partagée de la protection de la nature et du développement durable.

\section{BIBLIOGRAPHIE}

BANQUE MONDIALE \& UN-Water (2002), United Nations mechanism for inter-agency coordination on water resources, http://www.unwater.org/statistics.html. Consulté le 18 septembre 2012.

CNRS (2009), L'eau douce : une ressource précieuse, dossier Sagascience du CNRS, http:// www.cnrs.fr/cw/dossiers/doseau/. Consulté le 10 septembre 2012.

DE ROSA C. \& LYON J. (1997), Golden Dreams, Poisoned Streams. Mineral Policy Center, Washington DC.

FIDH (2007), Mali : l'exploitation minière et les droits humains, Mission internationale d'enquête, http:// www.fidh.org. Consulté le 13 septembre 2012.

FODE-MOUSSA K. (2006), Activités des entreprises minières canadiennes à l'étranger et leurs impacts sur les communautés, http://www.ieim.uqam.ca/IMG/pdf/MKeita_Texte_Formation_AQOCI.pdf.

Consulté 13 septembre 2012.

GALDIBÉ G. (2008), Étude pétrographique et structurale de la carrière de Bergui-Bidzar, Mémoire de Maîtrise, Université de Ngaoundéré.

GAVRILIUK V.G. \& BERNS H. (1999), High nitrogen steels : structure, properties, manufacture, applications, Springer,

http://www.miningwatch.ca/index.php?/Newsletter_7/Mining_Water_Pollution. Consulté le 12 septembre 2012 .

HERRINGTON (2008), Déclaration, http://www.miningwatch.ca. Consulté le 13 septembre 2012.

LEMIEUX E. (2010), Pérou : défis du développement local en région minière, Fondation Walter et Duncan Gordon (en ligne), Consulté le 13 septembre 2012.

LINGUE A. (2012), Les populations riveraines revendiquent les redevances dues à l'exploitation minière à Figuil, Cameroun Tribune, 18 septembre. 
LYON J. (1997), Interview, Mineral Policy Center, Washington DC.

MINING WATCH CANADA (2006), EMCBC Mining and the Environment primer : Acid Mine Drainage, http://www.miningwatch.ca/emcbc-mining-and-environment-primer-acid-mine-drainage. Consulté le 05 février 2010.

MINING WATCH (2001), Mining and Water Pollution in Canada, http://www.miningwatch.ca/updir/ amd.pdf. Consulté le 13 septembre 2012.

MINING WATCH (2000), Environmental mining council of bc : acid mine drainage : mining \& water pollution, ISSUES IN BC, http://dwb4.unl.edu/Chem/CHEM869R/CHEM869RLinks/ emcbc.miningwatch.org/emcbc/library/amd_water.htm. Consulté le 13 septembre 2012.

MIRANDA M., CHAMBERS D. \& COUMANS C. (2005), http://

www.frameworkfcorresponsiblemining.org/. Consulté le 13 septembre 2012.

ONU (2012), L'eau, une responsabilité partagée, deuxième rapport mondial sur la mise en valeurs des ressources en eau, http://unesdoc.unesco.org/images/0014/001444/144409F.pdf. Consulté le 19 décembre 2013.

ROCAGLIA (2008), Documents de synthèse des réalisations sociales, $27 \mathrm{p}$.

TOUMBA O. (2009), Dynamique des paysages du piémont de Wapouzé-Bidzar, Mémoire de Master, Université de Ngaoundéré.

UNEP (2000), L'Avenir de l'environnement mondial, http://www.unep.org/Geo2000/ov-fr.PDF. Consulté le 12 septembre 2012.

VEYRET Y. \& PECH P. (1992), L'homme et l'environnement, Paris, PUF, 424 p.

WAKPONOU A., MAINGUET M. \& DUMAY F. (2007), « L'homme et l'arbre dans les aires sèches du Nord-Cameroun ", Journal algérien des régions arides, 6, pp. 5-17.

WATIO ZEBABOU D. (2008), Gestion environnementale de l'entreprise CIMENCAM, Rapport de fin de stage, ESG-Douala.

WORLD GOLD COUNCIL (1996), www.safewater.org. Consulté le 12 septembre 2012.

\section{NOTES}

1. CIMENCAM, Les Cimenteries du Cameroun, filiale du groupe Lafarge, de renommée mondiale, dont le siège social au Cameroun se trouve à Douala.

2. Source : Rapports consolidés 2009, 2010 et 2011 de l'ITIE Cameroun 2013.

3. Source : Archives hospitalières de Figuil et du dispensaire de Bidzar.

4. Données issues des malades enregistrés dans les archives du dispensaire de Bidzar et de l'Hôpital de Figuil et du rapport du $3^{\mathrm{e}} \mathrm{RGPH}$ au Cameroun.

5. Source : Délégation régionale MINMIDT.

6. D'après la classification de Ramade (1981), basée sur une grille de qualité des eaux fondée notamment sur les propriétés chimiques.

7. Source: Poste forestier de Figuil et documents de synthèse des réalisations sociales de l'entreprise ROCAGLIA, 2008.

8. Source : Poste forestier de Figuil.

9. Rapport 2009 CIMENCAM

10. Alejandro Sánchez, Directeur des relations publiques de la mine Lagunas Norte, appartenant au géant minier canadien Barrick Gold, située dans le département de La Libertad au Pérou. 


\section{RÉSUMÉS}

La transformation du paysage végétal, ainsi que la pollution des nappes phréatiques et de l'air que connaît l'arrondissement de Figuil à cause de l'exploitation minière, l'expose à un risque écologique. Déjà, le taux de morbidité des populations est assez alarmant. Pour faire l'état des lieux de ces phénomènes, nous avons procédé, d'une part, à des enquêtes socioenvironnementales portant sur la santé des populations, les actions environnementales des entreprises minières et à la quantification de la pollution de l'air atmosphérique ; et, d'autre part, à des analyses chimiques des échantillons d'eau des puits et forages. L'étude révèle une dégradation d'un environnement déjà fragile et une aggravation de la crise de santé. On constate aussi que le nouveau code minier en vigueur, qui met l'accent sur la protection de l'environnement et la prise en compte des droits des riverains, tarde à apporter les effets escomptés. Ainsi, au-delà du constat de l'importance de l'enjeu de l'exploitation minière, il est nécessaire d'initier une dynamique d'action qui passe avant tout par l'affichage de stratégies d'actions motivées, non plus par des «coups médiatiques» (pollutions accidentelles, discours alarmistes des médias, revendications calculées des ONG), mais plutôt, par la mise en œuvre des exigences réglementaires et une prise en compte des demandes socio-environnementales.

The transformation of vegetal landscape, groundwater and atmospheric air pollutions of Figuil subdivision due to mining expose the region to ecological risk. Above all, the morbidity rate in this area is alarming. To evaluate the state of limestone exploitation effects, we have carried out, on one hand, socio-environmental studies on the health of inhabitants, environmental actions of the enterprises and the evaluation of atmospheric air pollution. On the other hand, we did the chemical analysis of samples of water from wells and bore-holes. The study reveals the environmental degradation of the already fragile milieu and the rise of health crises. In addition, the Cameroon's mining code has been revised to take into consideration the environmental protection and the rights of the local populations, but we observed that the current mining code takes a long time to be implemented. Beyond the report drawn up on mining exploitation, there is a lot at stake, it is necessary to initiate a dynamic action by displaying the strategies of motivated actions not by media frame-up (accidental pollutions, alarming media speeches, the NGOs' partial reports) but by the regulation and socio-environmental requirements.

\section{INDEX}

Mots-clés : dégradation, environnement, crise de santé, Figuil, Cameroun

Keywords : environment, health crisis, Cameroon

\section{AUTEURS}

\section{OUMAROU TOUMBA}

Assistant Lecturer, Department of Geography, FSMS, Université de Buea (Cameroun), ttoumba@yahoo.com 
ANSELME WAKPONOU

Assistant Lecturer, Department of Geography, FSMS, Université de Buea (Cameroun), wakponouanselme@yahoo.fr 